\title{
Effect of additional inhibition of human epidermal growth factor receptor 2 with the bispecific tyrosine kinase inhibitor AEE788 on the resistance to specific EGFR inhibition in glioma cells
}

\author{
SABINA BEREZOWSKA ${ }^{1,6}$, SIMONE DIERMEIER-DAUCHER ${ }^{2}$, GERO BROCKHOFF $^{2}$, RAYMONDE BUSCH $^{3}$, \\ JUSTUS DUYSTER $^{4}$, ANCA-LIGIA GROSU ${ }^{5,7}$ and JÜRGEN SCHLEGEL ${ }^{1}$ \\ ${ }^{1}$ Division of Neuropathology, Institute of Pathology, Technische Universität München, Trogerstr. 18, D-81675 Munich; \\ ${ }^{2}$ Department of Obstetrics and Gynecology, University of Regensburg, Landshuter Str. 65, D-93059 Regensburg; ${ }^{3}$ Institute of \\ Medical Statistics and Epidemiology; ${ }^{4}$ Department of Internal Medicine III; ${ }^{5}$ Department of Radiation Oncology, \\ Klinikum Rechts der Isar, Technische Universität München, Ismaninger Str. 22, D-81675 Munich, Germany
}

Received April 21, 2010; Accepted June 28, 2010

DOI: $10.3892 /$ ijmm_00000518

\begin{abstract}
Targeted molecular therapies against the epidermal growth factor receptor (EGFR) are novel, promising and potentially radiosensitising therapeutic approaches in the treatment of glioblastoma, a highly malignant and treatmentrefractory brain tumour. Despite a solid rational basis, specific EGFR inhibition has rendered only disappointing clinical results to date. We therefore evaluated the efficacy of additional inhibition of human epidermal growth factor receptor 2 (HER2), the 'non-autonomous amplifier' of EGFR signalling. Glioblastoma cells (LN-18, LN-229) with different co-expression levels of EGFR and HER2 were treated with specific EGFR and bispecific EGFR/HER2 tyrosine kinase inhibitors (TKIs) (AG1478, AEE788) and experimental radiotherapy, followed by assessment of growth inhibition. Activity of the major downstream signalling pathways Akt and MAPK was determined by immunoblotting. EGFR-overexpressing LN-18 cells $\left(\mathrm{EGFR}^{+++} / \mathrm{HER} 2^{+}\right)$showed resistance and HER2-overexpressing LN-229 cells (EGFR ${ }^{+} / \mathrm{HER}^{++}$) showed sensitivity to EGFR-specific inhibition. Interestingly, resistance of LN-18 to EGFR inhibition was overcome by AEE788 treatment, supposedly due to its additional HER2 inhibition. Application of AEE788 resulted in blockage of EGF-dependent EGFR/HER2-heterodimer activation in LN-18 cells, disclosing a possible mediating mechanism for overcoming EGFR-resistance. TKI treatment resulted in significant
\end{abstract}

Correspondence to: Dr Sabina Berezowska, ${ }^{6}$ Present address: Institute of Pathology, Ludwig-Maximilians-University, Thalkirchner Str. 36, D-80337 Munich, Germany

E-mail: sabina.berezowska@med.uni-muenchen.de

Present address: ${ }^{7}$ Department of Radiation Oncology, University of Freiburg, Robert-Koch-Str. 3, D-79106 Freiburg, Germany

Key words: glioblastoma, targeted therapy, epidermal growth factor receptor, HER2/neu blockage of both Akt and MAPK signalling pathways, but an incomplete inhibition of PI3K/Akt paralleled the resistance of cells to TKI-induced growth inhibition. Furthermore, the bispecific EGFR/HER2 inhibitor AEE788 showed a radiosensitising effect in EGFR-overexpressing cells. Taken together, we conclude that inhibition of HER2 in EGFRoverexpressing tumours may harbour the potential to overcome resistance to EGFR-targeted therapy and exert radiosensitising properties. We suggest that responsiveness to EGFR targeted therapy is mediated through impairment of EGFR/ HER2 heterodimer signalling, and thus depends on the ratio of EGFR to HER2 rather than on the amount of individual receptors.

\section{Introduction}

Glioblastoma (GBM) is the most frequent brain tumour and among the most malignant of all human neoplasms, with dismal patient outcome. In retrospective population-based studies from Switzerland and Canada, the median survival time for all patients is 4.9 months; $<20 \%$ of patients survived $>1$ year, and $<3 \%$ survived $>3$ years $(1,2)$. It is one of the most treatment-refractory tumours and is highly resistant to current standard therapies (i.e., surgery, radiotherapy and chemotherapy). If feasible, maximal surgical resection improves survival, but complete resection is virtually impossible due to the highly infiltrating nature of the neoplasm. Despite all efforts to improve radiation and chemotherapy protocols, the prognosis of patients has changed very little to date (3). Therefore novel therapeutic agents and approaches are exceptionally desirable.

Novel and highly promising oncological therapies currently under development target and inhibit the activities of specific molecules that contribute to the malignancy and radioresistance of malignant neoplasms. This approach has already resulted in valuable clinically approved pharmacological agents. Perhaps the first success story is a selective inhibitor of the ABL tyrosine kinase (Imatinib mesylate/ Glivec ${ }^{\circledR}$ ), which proved effective in putting nearly all patients with BCR-ABL-driven chronic-phase CML into complete remission (4). This proof 
of concept edified the image of 'oncogenic addiction', the dependence of tumour cells on specific molecular events (5-7).

The rationale for targeting the ErbB or epidermal growth factor receptor (EGFR) family of protein tyrosine kinases in GBM is compelling, as they are known to contribute to malignant processes (8), and overexpression of EGFR presents a key signalling pathway in de novo GBM (9). ErbB receptor signalling is induced by ligand dependent homo- or heterodimerisation of individual receptor monomers [EGFR (ErbB1), HER2 human epidermal growth factor receptor 2 (HER2, ErbB2), HER3 (ErbB3) and HER4 (ErbB4)], which triggers signal transduction mainly through the PI3K/Akt- and MAPKpathways (8). HER2 does not interact with extracellular ligands, but forms a basal level of homodimers due to the constitutive extension of its dimerisation loop. Most notably, however, HER2 acts as a 'non-autonomous amplifier' (10), it serves as the preferred dimerisation partner for the other ErbB receptors, with signalling potency of HER2-heterodimers markedly exceeding those of receptor-homodimers $(8,10)$. Interestingly, in a recent study, co-expression of HER2 was observed in the majority of GBM specimens (>80\%), with high expression patterns detectable in de novo GBM (11). Supposedly due to tumour heterogeneity, other studies rendered lower expression patterns for HER2, but most of them showed co-expression of EGFR and HER2 in the majority of GBM specimens (12-16).

One method to inhibit ErbB receptors is the administration of specific small-molecule tyrosine kinase inhibitors (TKIs), cell-permeable agents that compete with ATP at the catalytic domain of the protein tyrosine kinase, thereby inhibiting transphosphorylation of the receptor and downstream signalling. Several EGFRs targeting TKIs proved effective in other solid cancers (17), but showed only very disappointing effects in GBM $(18,19)$. In a recent study, the addition of Erlotinib, a selective EGFR inhibitor, to current standard glioblastoma therapy with Temozolomide and radiation did not show any improvement in overall survival, nor did analyses for EGFR, the truncated EGFR EGFRvIII, p53, phosphatase and tensin homolog (PTEN), combination EGFR and PTEN, and EGFR amplification status predict sensitivity to EGFR inhibition (19). The failure to predict sensitivity to EGFR-inhibitors from EGFR protein expression may be due to several reasons. Keeping in mind the extensive cross-talk between the ErbB receptor family members, presumably the most important cause is additional tumour dependence on other receptor protein kinases, providing a rationale for concomitant multi-receptor inhibition.

In the present study, we aimed to elucidate the additional receptor dependence of EGFR-TKIs in a preclinical experimental setting. Targeting cell lines expressing an approximately inverse ratio of EGFR and HER2 (LN-18 and LN-229) with specific EGFR- and bispecific EGFR/HER2inhibiting TKIs (AG1478 and AEE788), we explored the importance of HER2-signalling in GBM and evaluated the major downstream signalling pathways. As ErbB receptors are known to mediate radioprotective cellular effects through Akt and MAPK-signalling (20) and account for at least part of 'accelerated repopulation' (i.e., enhanced cellular proliferation after exposure to ionizing radiation) (21), we finally evaluated the radiosensitising property of TKIs.

\section{Materials and methods}

Cell cultivation. Cell lines LN-18 and LN-229 derived from human de novo GBM $(22,23)$ were maintained as monolayers in Dulbecco's modified Eagle medium (DMEM) supplemented with $10 \%$ foetal bovine serum (FBS), $1 \%$ L-glutamate, $100 \mathrm{U} / \mathrm{ml}$ penicillin and $100 \mu \mathrm{g} / \mathrm{ml}$ streptomycin (Gibco-BRL, Karlsruhe, Germany) in a humidified atmosphere at $37^{\circ} \mathrm{C}$ and $5 \% \mathrm{CO}_{2}$.

Inhibitors. Tyrphostin AG1478 (Sigma-Aldrich, Irvine, UK) is known to be a highly specific EGFR TKI [ $\mathrm{IC}_{50}(\mathrm{EGFR})=$ $0.003 \mu \mathrm{M} ; \mathrm{IC}_{50}($ HER 2$\left.)>100 \mu \mathrm{M}\right](24)$. The pyrrolopyrimidine AEE788 (kindly provided by Novartis Pharma AG, Basel, Switzerland) represents a bispecific EGFR-/HER2-TKI $\left[\mathrm{IC}_{50}(\mathrm{EGFR})=0.002 \mu \mathrm{M}, \mathrm{IC}_{50}(\right.$ HER2 $\left.)=0.006 \mu \mathrm{M}\right)$, with additional potency to inhibit VEGFR2 $\left(\mathrm{KDR} / \mathrm{Flk}-1\right.$; $\mathrm{IC}_{50}=$ $0.077 \mu \mathrm{M})$ (25). Specific inhibition of the two major downstream signalling pathways was achieved using the specific PI3-kinase inhibitor LY294002 (Calbiochem-Novabiochem, Schwalbach, Germany) $\left[\mathrm{IC}_{50}(\mathrm{PI} 3 \mathrm{~K})=1.4 \mu \mathrm{M} ; \mathrm{IC}_{50}(\mathrm{MEK})>\right.$ $50 \mu \mathrm{M}]$ (26), and PD98059 (Calbiochem-Novabiochem), a selective inhibitor of the MAP kinase activating enzyme MEK $\left[\mathrm{IC}_{50}(\mathrm{PI} 3 \mathrm{~K})>100 \mu \mathrm{M} ; \mathrm{IC}_{50}(\mathrm{MEK})=2 \mu \mathrm{M}\right](27,28)$. The selectivity of all inhibitors was confirmed in cell-based assays (24-26,28). Inhibitors were dissolved and used according to the manufacturer's specifications.

Western blot analysis. Protein extraction from cultured cells was accomplished using lysis buffer containing $20 \mathrm{mM}$ Tris$\mathrm{HCl}$ (pH 7.5), $150 \mathrm{mM} \mathrm{NaCl}, 1 \mathrm{mM}$ EDTA, 1 mM EGTA, $1 \%$ Triton $\mathrm{X}-100,2.5 \mathrm{mM}$ sodium pyrophosphate, $1 \mathrm{mM}$ sodium orthovanadate, $1 \mathrm{mM} \beta$-glycerolphosphate, $1 \mu \mathrm{g} / \mathrm{ml}$ leupeptin and $1 \mathrm{mM}$ PMSF.

If cells were treated before lysis, they were serum starved for $12 \mathrm{~h}$, followed by incubation with inhibitors in the concentrations indicated for $1 \mathrm{~h}$ and addition of $10 \mathrm{ng} / \mathrm{ml}$ EGF (Upstate Biotechnology, NY, USA) for $5 \mathrm{~min}$.

Lysates were clarified by centrifugation and their protein concentrations were determined using the Bradford reagents according to the manufacturer's specifications (Bio-Rad Laboratories Inc., Munich, Germany).

Equal amounts of protein were resolved on sodium dodecyl sulphate-polyacrylamide gel electrophoresis (SDS-PAGE), transferred to polyvinylidene difluoride (PVDF) membranes (Immobilion Millipore Corporation, Bedford, USA) and incubated with the specific primary antibodies, rabbit polyclonal anti-EGFR $(1: 1,000$; Santa Cruz Biotechnology, Inc., Santa Cruz, CA, USA), rabbit polyclonal anti-HER2 (1:500; DakoCytomation, Glostrup, Denmark), rabbit polyclonal antiAkt $(1: 1,000)$, rabbit polyclonal anti-pAkt (Ser473; 1:1,000), rabbit polyclonal anti-p42/44 MAPK $(1: 1,000)$, rabbit polyclonal anti-pp42/44 MAPK (Thr202/Thr204; 1:1,000) and the respective goat polyclonal anti-rabbit and sheep polyclonal antimouse horseradish peroxidase (HRP)-conjugated secondary antibody $(1: 2,000$; unless otherwise indicated, all antibodies were from Cell Signaling Technology Inc., Beverly, MA, USA). Where necessary, the mouse monoclonal anti- $\alpha$-tubulin antibody (Sigma-Aldrich) was applied to stain $\alpha$-tubulin as a loading control. 
Blots were developed using SuperSignal chemiluminescent substrates from Pierce (Perbio Science, Bonn, Germany). Densitometric band analysis was performed using ImageJ Software $1.37 \mathrm{v}$ (developed at the US National Institutes of Health and available on the Internet at http://rsb.info.nih. gov/ij/).

Immunoprecipitation. Following a 24-h deprivation from FBS, cells were treated in duplicate with 1 and $10 \mu \mathrm{M}$ AEE788 for $30 \mathrm{~min}$ before addition of $10 \mathrm{ng} / \mathrm{ml} \mathrm{EGF}$. Protein extraction was performed using lysis buffer containing $10 \mathrm{mM}$ Tris- $\mathrm{HCl}$ (pH 7.4), $130 \mathrm{mM} \mathrm{NaCl}, 5$ mM EDTA, 1\% Triton $\mathrm{X}-100,20 \mathrm{mM}$ sodium phosphate ( $\mathrm{pH} 7.5), 10 \mathrm{mM}$ sodium pyrophosphate $(\mathrm{pH} 7.0), 50 \mathrm{mM} \mathrm{NaF}, 1 \mathrm{mM}$ sodium orthovanadate, $1 \mathrm{mM}$ B-glycerolphosphate and protease inhibitors (Roche Diagnostics, Mannheim, Germany).

After clarification by centrifugation and preclearing with protein A-Sepharose (Amersham/Pharmacia Biotech, Freiburg, Germany), $5 \mu \mathrm{M}$ anti-EGFR antibody was added to the supernatant. The addition of an anti-mouse antibody (Jackson ImmunoResearch, Baltimore) to one probe served as a negative control for unspecific binding. Antibody-protein complexes were precipitated with protein A-Sepharose. For immunoblotting control, whole cell lysates and bound fractions, respectively, were subjected to SDS-PAGE and blotted on PVDF membranes. Detection of phosphotyrosine was performed using a mixture of the antiphosphotyrosine monoclonal mouse antibodies 4G10 (1:2,000; Upstate Biotechnology) and pY20 (1:2,000; PharMingen, Heidelberg, Germany). After incubating blots with HRP-conjugated secondary antibody $(1: 10,000$; polyclonal sheep anti-mouse and donkey anti-rabbit, respectively, Amersham/Pharmacia Biotech) they were developed using SuperSignal chemoluminescent substrates from Pierce (Perbio Science). Subsequently, the antibodies were stripped and the membranes reprobed with anti-EGFR or anti-HER2 antibodies.

Flow cytometry. Freshly harvested cells were transferred to ice and kept at $0^{\circ} \mathrm{C}$ in the dark during the entire labelling procedure. Immediately after detachment, cells were washed twice in ice-cold washing buffer [phosphate buffered saline (PBS), pH 7.4, supplemented with $0.2 \%$ bovine serum albumin (BSA) and $0.01 \% \mathrm{NaN}_{3}$ ]. A total of $5 \times 10^{5}$ cells were centrifuged and resuspended in $40 \mu \mathrm{l}$ of labelling solution consisting of $20 \mu 1 \mathrm{PAB}$ (PBS, pH 7.4, supplemented with $2 \% \mathrm{BSA}$ and $0.01 \% \mathrm{NaN}_{3}$ ) and $20 \mu \mathrm{l}$ of the antibodies for target labelling. For EGFR and HER2 labelling, cells were incubated with PE-conjugated anti-EGFR antibody (clone EGFR.1, subclass IgG2b, BD Biosciences, San Jose, CA, USA) or PE-conjugated anti-HER2 antibody (clone Neu 24.7, subclass IgG1, BD Biosciences). After $30 \mathrm{~min}$ of lightprotected incubation on ice, cells were washed twice in washing buffer and analyzed on a FACS Calibur flow cytometer (BD Biosciences). PE was excited with a $488 \mathrm{~nm}$ aircooled argon-ion laser $(15 \mathrm{~mW})$ and measured on detection channel 2 with a 585/42 nm bandpass filter. Sample acquisition (20,000 events per sample) was performed with CellQuest Pro Software (BD Biosciences) using a Macintosh G4 computer. Live cells were gated in an FSC (forward scatter)/ SSC (sideward scatter) dot blot with FlowJo Software (Tree
Star Inc., Ashland, OR, USA) and used for quantification of PE fluorescence intensity in a histogram. Unlabelled and PElabelled samples were used to determine the mean background fluorescence and the mean fluorescence intensity (MFI) in the PE (585/42 nm)-channel, respectively. Data are presented as fraction of MFI/background.

Crystal violet cell quantification assay. Cells were plated onto 24 -well dishes at $2 \times 10^{4}$ cells/well, treated with a single administration of inhibitors. The assay terminated up to $48 \mathrm{~h}$ after treatment by fixation with $4 \%$ formaldehyde in PBS for 30 min. After washing with PBS containing $0.1 \%$ Triton X-100 and washing with deionised water, cells were stained with crystal violet $(0.04 \%$ crystal violet in $4 \%(\mathrm{v} / \mathrm{v})$ ethanol; $500 \mu \mathrm{l} /$ well for $30 \mathrm{~min}$ ) and subsequently washed with deionised water. In order to solubilise the nucleoproteinbound crystal violet, $500 \mu \mathrm{l}$ of $1 \%$ SDS solution was added to each well and incubated on a shaker for $1 \mathrm{~h}$. The solution was transferred to 96-well dishes (100 $\mu \mathrm{l} /$ well), and absorbance was measured at $570 \mathrm{~nm}$. Linearity between cell count and absorbance for the range of cell numbers tested was confirmed in preliminary experiments applying Pearson's linear correlation analysis $\left[\mathrm{p}<0.0001 ; \mathrm{R}^{2}(\mathrm{LN}-18)=0.94 ; \mathrm{R}^{2}(\mathrm{LN}-229)=\right.$ 0.93] (data not shown).

For evaluation of cytotoxicity, either the absorbance measurement values were plotted directly as arbitrary units or cell growth in each well was calculated as the percentage of the average absorbance of the untreated control.

Colony formation assay. The effects of bimodal treatment (TKI/irradiation) were measured by assessing the colony formation ability of cells, a method representing the radiobiologic gold standard (29).

Cells were plated into $24-w e l l$ dishes at $2 \times 10^{4}$ cells/well and treated with a single administration of $5 \mu \mathrm{M}$ AG1478 or AEE788. After $12 \mathrm{~h}$, cells were $\gamma$-irradiated with single doses of $0-10$ Gy using a cobalt ${ }^{60} \mathrm{Co}$ source $(6 \mathrm{MeV})$ at a dose rate of $6 \mathrm{~Gy} / \mathrm{min}$.

Cells were harvested $48 \mathrm{~h}$ after inhibitor-treatment. They were then diluted (1:200) and plated into 6-well dishes. After a 10-day incubation, cells were fixed and stained using DiffQuik reagents (Medion Diagnostics AG, Düdingen, Switzerland). A flat bed scanner was used to acquire RGB colour images of the culture dishes at a resolution of 300 pixels per inch (ppi), which were converted to grey-scale images, followed by automated counting of colonies by image analysis (30). Colonies consisting of $>50$ cells were counted. The surviving fraction was calculated as the percentage of colonies relative to controls.

Surviving fractions determined with the colony formation assay were fitted into the classical linear-quadratic equation, $\mathrm{S}=\exp \left(-\alpha \mathrm{D}-\beta \mathrm{D}^{2}\right)$, where $\mathrm{S}$ is the surviving fraction, $\mathrm{D}$ the radiation dose and $\alpha$ and $\beta$ adjustable parameters (29). The $95 \%$ confidence band was plotted for each survival curve. Calculations were made through nonlinear least-squares regression using GraphPad Prism software, version 5 (GraphPad Software Inc, La Jolla, CA, USA).

Statistical analysis. Data analysis was performed using ImageJ Software $1.37 \mathrm{v}$ (US National Institutes of Health), SPSS 
software version 15 (SPSS Inc, Chicago, IL, USA) and GraphPad Prism software, version 5 (GraphPad Software Inc.). The statistical tests used are indicated in figure legends. Significance level was set to 0.05 .

\section{Results}

Inverse ratio of EGFR and HER 2 oncoprotein co-expression in $L N-18$ and $L N-229$ glioma cell lines. As the relative expression of HER2 is known to change depending on culture conditions (31), a quantitative expression profile of EGFR and HER2 was performed for the present experimental conditions using FACS-analysis and immunoblotting. We found that the glioma cell lines LN-18 and LN-229 express an approximately inverse ratio of EGFR and HER2, respectively [LN-18 (EGFR ${ }^{+++}$/ HER2 $\left.^{+}\right)$, LN-229 $\left(\right.$EGFR $\left.^{+} / \mathrm{HER}^{++}\right)$] (Fig. 1). In both cell lines the difference between EGFR and HER2 expression was highly significant $(\mathrm{p}<0.001)$ (Fig. 1A).

Effect of the bispecific EGFR/HER2 tyrosine kinase inhibitor AEE788 on EGFR/HER2 heterodimer signalling in glioma cell lines. As shown in earlier studies, AEE788 acts as a bispecific inhibitor of EGFR and HER2 (25). To elucidate its biological effect on glioma cells, we first verified its impact on EGFR and HER2 signalling. Representative serum-starved LN-18 cells were treated with increasing concentrations of AEE788 and EGF, followed by immunoprecipitation of EGFR. Receptor-activation (i.e., phosphorylation) was then visualised by strong phosphotyrosine immunoreactivity and was found to be EGF-dependent (Fig. 2, upper panel). The absence of phosphotyrosine immunoreactivity represented the potency of AEE788 to block receptor activation (Fig. 2, upper panel). Of interest, HER2 detection in the immunoprecipitated lysate (Fig. 2, lower panel) suggested pronounced EGFR/HER2-heterodimer formation, consistent with HER2 being the preferred dimerisation partner for all ErbB receptors (8). Stimulation with EGF produced marked receptor phosphorylation and downregulation of total EGFR and HER2 (Fig. 2, second row), a phenomenon known to take place following receptor activation (8).

Taken together, we have shown that $1 \mu \mathrm{M}$ AEE788 (32) causes complete inhibition of EGF-induced EGFR and EGFR/HER2 activation in LN-18 cells and furthermore impairs receptor downregulation, as indicated by strong protein bands of total EGFR and HER2. EGF-dependent EGFR/HER2 heterodimer formation was found to remain unchanged following AEE788 treatment (Fig. 2, middle and lower panel); however, the activation (phosphorylation) of EGFR/HER2 heterodimers was clearly inhibited (Fig. 2, upper panel). Similar results were found for LN-229 cells by Failly et al (32).

Growth-inhibiting effects of AEE788 in LN-18 and LN-229 glioma cell lines. Kinetic experiments with increasing concentrations of AEE788 $(0.1-25 \mu \mathrm{M})$ were conducted in both cell lines, evaluating its growth inhibiting effect at up to $48 \mathrm{~h}$ following single dose treatment (Fig. 3).

In LN-18 cells, a minor, though significant growthinhibition induced by $1 \mu \mathrm{M}$ AEE788 was assessed at $48 \mathrm{~h}$ treatment only $(\mathrm{p}<0.001$; as compared to the untreated control), whereas $5 \mu \mathrm{M}$ AEE788 exerted a marked growth-inhibiting effect ( $\mathrm{p}<0.05$ at $12 \mathrm{~h}, \mathrm{p}<0.001$ at $18-48 \mathrm{~h}$ ) (Fig. 3A, upper
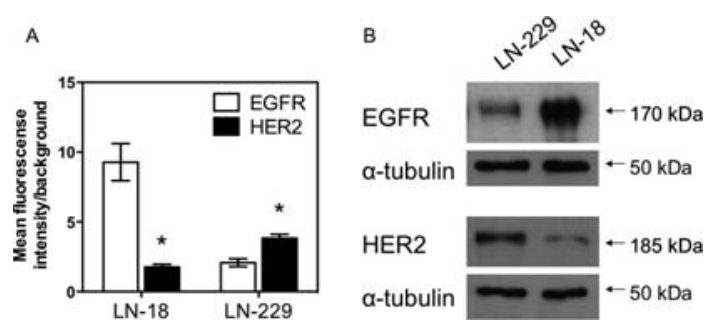

Figure 1. EGFR and HER2 expression in LN-18 and LN-229 cells as detected through (A) FACS-analysis and (B) Western blotting. (A) Cells were labelled with PE-conjugated anti-EGFR or anti-HER2 antibody and analysed by flow cytometry. Cells were gated in an FSC/SSC dot blot and PE fluorescence intensity was quantified in a histogram. Unlabelled and PE-labelled samples were used to determine the mean background fluorescence and the mean fluorescence intensity (MFI). Data are presented as fraction of MFI/ background (columns), and error bars represent the SD of three independent experiments. *Significant difference of EGFR and HER2 expression in LN-18 and LN-229 cells as determined with a two-way ANOVA and Bonferroni post-hoc test ( $\left.{ }^{\mathrm{p}} \mathrm{p}<0.001\right)$. (B) The receptor status of $\mathrm{LN}-18$ and LN-229 was confirmed by immunoblotting using anti-EGFR and anti-HER2 antibodies. To ensure equivalent loading and transfer, an $\alpha$-tubulin-control was conducted.

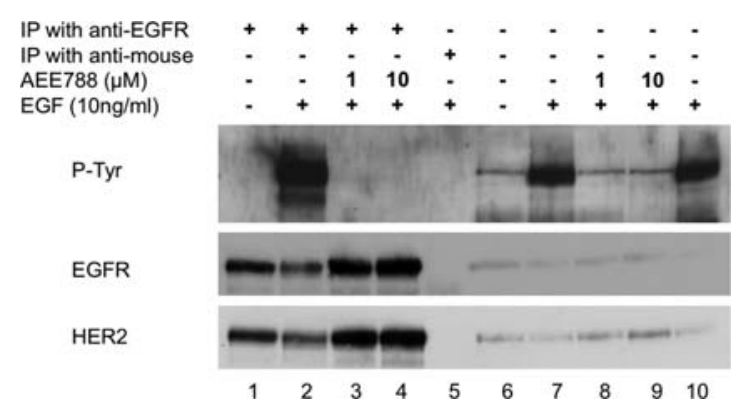

Figure 2. Phosphorylation/activation of EGFR and HER2 (upper panel), EGFR expression (middle panel) and HER2 expression (lower panel) after AEE788 treatment and immunoprecipitation (IP) of EGFR in LN-18 cells. LN-18 cells were treated with $\operatorname{AEE} 788(1,10 \mu \mathrm{M})$ and epidermal growth factor (EGF, $10 \mathrm{ng} / \mathrm{ml}$ ) as indicated. SDS gels were loaded in duplicate, each probed for phosphotyrosine (p-Tyr; upper panel), stripped and subsequently probed for EGFR (middle panel) and HER2 (lower panel), respectively. Protein-lysates immunoprecipitated with anti-mouse antibody (line 5) and non-immunoprecipitated whole cell lysates (line 6 to 10) served as control.

panel). In LN-229 cells, all applied dosages starting at $0.1 \mu \mathrm{M}$ AEE788 resulted in marked growth inhibition $(\mathrm{p}<0.001$ at $12,18,24,36$ and $48 \mathrm{~h}$; as compared to the untreated control), and there was no significant difference between 1 versus $5 \mu \mathrm{M}$ AEE788 (Fig. 3A, lower panel). In both cell lines, 10 and $25 \mu \mathrm{M}$ AEE788 were shown to act in an excessively cytotoxic manner, possibly due to a loss of specificity for EGFR and HER2 inhibition.

Different reaction of $L N-229$ and $L N-18$ cells to EGFRspecific (AG1478) versus bispecific EGFR/HER2 inhibition (AEE788). In the kinetic experiments stated above, $5 \mu \mathrm{M}$ AEE788 exerted marked, yet not overshooting growthinhibiting effects in both cell lines compared to untreated controls. To elucidate whether these effects were mediated through specific EGFR inhibition, cells were exposed to equimolar doses of AG1478 and AEE788, and the assay was terminated $48 \mathrm{~h}$ later by staining with crystal violet. 

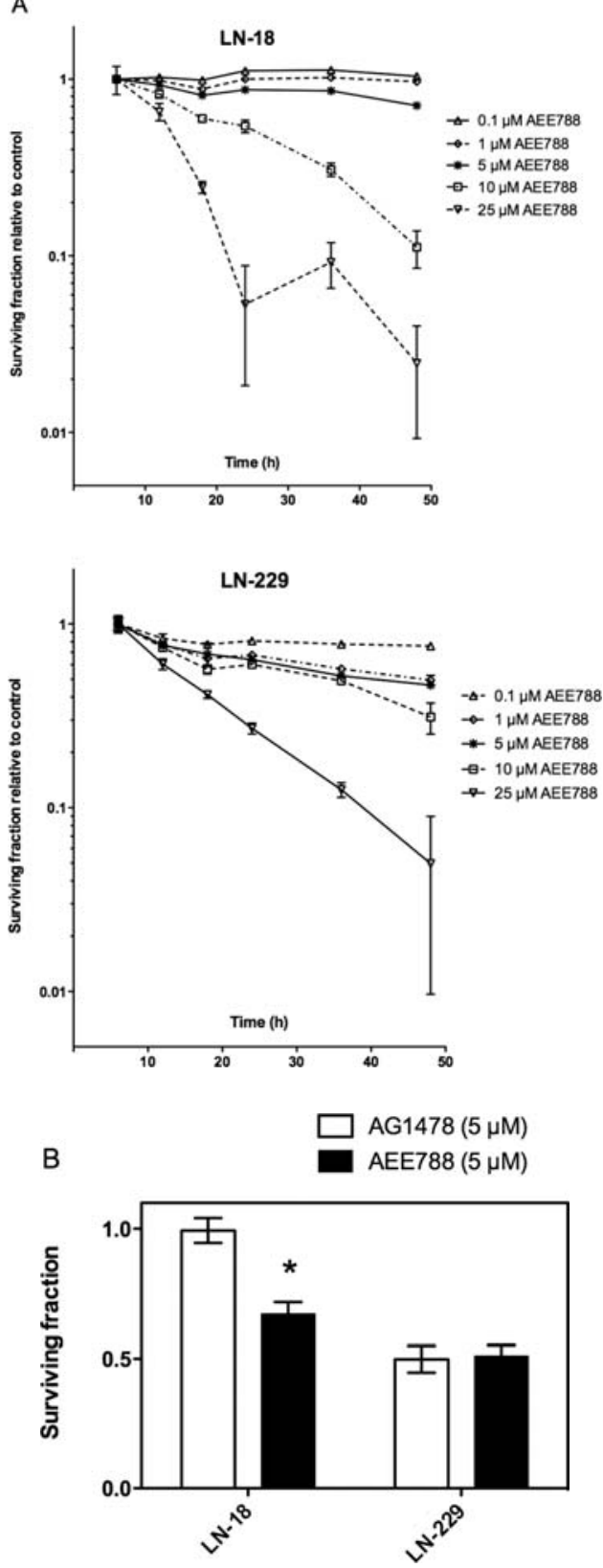

Figure 3. Growth-inhibiting effect of specific EGFR inhibition (AG1478) versus bispecific EGFR/HER2 inhibition (AEE788) in LN-18 and LN-229 cells. (A) In both cell lines, $5 \mu \mathrm{M}$ AEE788 showed significant growthinhibiting effects compared to untreated control, yet avoiding overshooting response. Cells were treated with increasing concentrations of AEE788 as indicated, and growth inhibition was evaluated 6, 12, 18, 24, 36 and $48 \mathrm{~h}$ after. Data points represent the survival fraction in percentage of the untreated control versus time. Error bars depict the SD of eight replicate values. The significance between the treatment groups at specific time points were determined with a two-way ANOVA and Bonferroni post-hoc test. Significant results were gained in LN-18 using $1 \mu \mathrm{M}$ AEE788 ( $<<0.001$ at $48 \mathrm{~h}), 5 \mu \mathrm{M}$ AEE788 (p<0.05 at $12 \mathrm{~h}, \mathrm{p}<0.001$ at $18-48 \mathrm{~h})$ and $10-25 \mu \mathrm{M}$ AEE788 ( $\mathrm{p}<0.001$ at $6-48 \mathrm{~h}$ ); in LN-229 using $0.1-25 \mu \mathrm{M}$ AEE788 ( $<<0.001$ at $12-48 \mathrm{~h}$ ). (B) Direct comparison of growth-inhibiting effects of $5 \mu \mathrm{M}$ AEE788 and $5 \mu \mathrm{M}$ AG1478 revealed a resistance of EGFR-overexpressing LN-18 cells to AG1478, which could be overcome by AEE788. Conversely, HER2-overexpressing cells were sensitive to sole EGFR inhibition (AG1478). Cells were incubated with $5 \mu \mathrm{M}$ AG1478 or AEE788 for $48 \mathrm{~h}$ and stained with crystal violet. The absorbance was measured at $570 \mathrm{~nm}$, and the surviving fraction was calculated as the percentage of control cell growth ( $0 \mu \mathrm{M}$ AG1478/AEE788). Error bars represent the SD of 24 replicate values. *Significance of the difference of growth-inhibition between AG1478 versus AEE788 treatment as determined with a two-way ANOVA and Bonferroni post-hoc test $\left({ }^{*} \mathrm{p}<0.001\right)$.
The results disclosed a different behaviour of LN-18 and LN-229 cells exposed to AG1478 or AEE788. LN-229 cells $\left(\mathrm{EGFR}^{+} / \mathrm{HER}^{++}\right)$reacted equally well to both compounds, showing a uniform decrease of the surviving fraction by $\sim 50 \%$ (Fig. 3B). Conversely, LN-18 cells (EGFR ${ }^{+++} / \mathrm{HER} 2^{+}$) responded in a clearly different fashion to treatment with AG1478 versus AEE788 ( $<<0.001)$. Sole EGFR inhibition with $5 \mu \mathrm{M}$ AG1478 had no significant effect compared to control $(\mathrm{p}>0.05)$, whereas $5 \mu \mathrm{M}$ AEE788 resulted in significant growth-inhibition $(\mathrm{p}<0.001)$, most likely due to additional HER2 inhibition (Fig. 3B).

In conclusion, we have shown the HER2-overexpressing cell line LN-229 to be relatively sensitive to sole EGFR inhibition. Most interestingly, however, the EGFR-overexpressing cell line LN-18 showed a relative resistance to sole EGFR inhibition, which could be overcome by treatment with AEE788, supposedly due to its additional HER2 inhibition.

Inhibition of EGFR and HER2 with TKIs and its effect on the Akt and MAPK signalling pathways in $L N-18$ versus $L N-229$ cells. Downstream effects of EGFR and EGFR/HER2 signalling are known to be mediated, in a large measure, by the MAPK and the PI3K/Akt pathways (8). Thus, it was of interest to investigate the effect of AG1478 and AEE788 on the activation of Akt and MAPK.

Immunoblot analyses revealed a similar TKI-induced reduction of phosphorylated (i.e., activated) MAPK in both cell lines, AEE788 proving more potent than specific EGFR inhibition using AG1478 in both cell lines (Fig. 4A and B). Thus, MAPK inhibition was found to be an important mediator of TKI treatment, but could not explain the differing behaviour of LN-18 versus LN-229 cells to sole EGFR inhibition.

In LN-229 cells phosphorylation of Akt was equally strongly impeded by both AG1478 and AEE788, even at low inhibitor dosages. Conversely, and most interestingly, Akt phosphorylation in LN-18 cells was blocked more efficiently by AEE788 than by AG1478. In addition, it was clearly dose-dependent (Fig. 4C). This appears to suggest that relative resistance of $\mathrm{LN}-18$ cells to the effects of AG1478 (specific EGFR inhibition) and its overcoming by AEE788 (additional HER2 inhibition) may, to some extent, be mediated through the Akt signalling pathway.

This finding was further explored by evaluating growthinhibiting effects induced by specific inhibition of the PI3K/ Akt-pathway by LY294002 versus the specific inhibition of the MAPK pathway by PD98059. Both cell lines reacted equally well to PI3K inhibition by LY294002, but LN-18 cells showed a relative resistance for the MEK inhibitor PD98059, paralleling the relative resistance of LN-18 cells to specific EGFR inhibition (AG1478) (data not shown). These results are in favour of the assumption that together with MAPK inhibition, PI3K/Akt-pathway inhibition may be crucial for the growth-inhibiting effect of TKIs.

Radiosensitising property of the bispecific EGFR/HER2 inhibitor AEE788 on LN-18 glioma cells. To examine whether receptor inhibition was able to alter radiation survival, $\mathrm{LN}-18$ and LN-229 cells were exposed to inhibitors with or without combined $\gamma$-irradiation with single doses of 2-10 Gy. The 


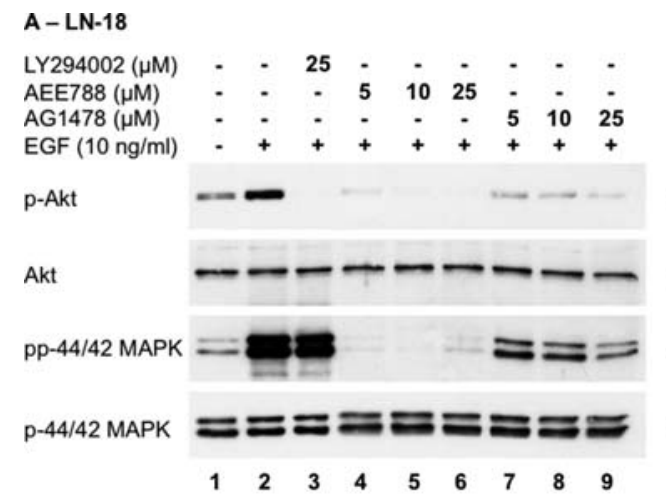

$40 \mathrm{kDa}$

$40 \mathrm{kDa}$

$44 / 42 \mathrm{kDa}$

$44 / 42 \mathrm{kDa}$

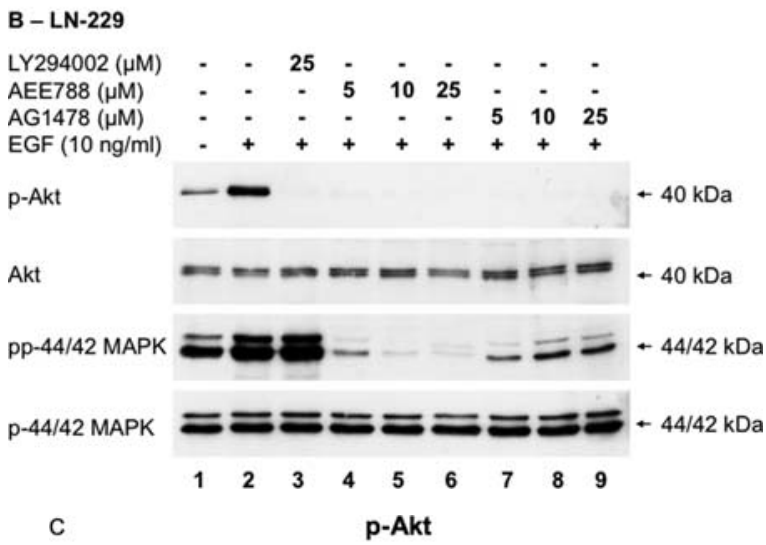

C

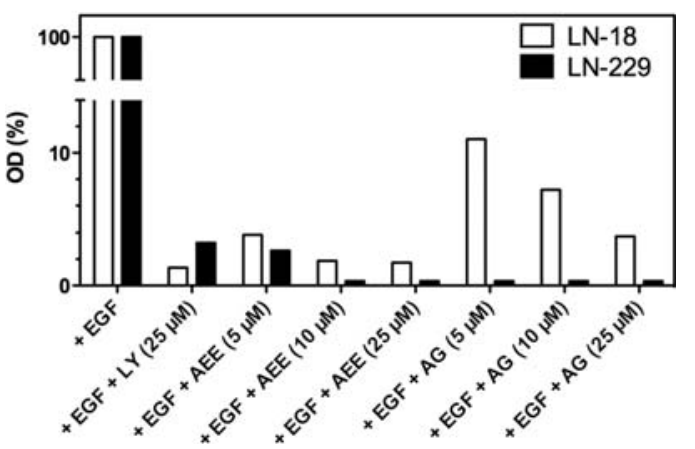

Figure 4. Effect of TKIs on the activation of Akt and MAPK in (A) LN-18 and (B) LN-229 cells. Cells were treated with LY294002 (25 $\mu \mathrm{M})$, AEE788 $(5,10,25 \mu \mathrm{M}), \operatorname{AG} 1478(5,10,25 \mu \mathrm{M})$ and epidermal growth factor (EGF, $10 \mathrm{ng} / \mathrm{ml}$ ) as indicated, and the protein extracts analyzed by immunoblotting. To ensure equivalent loading and transfer, blots were probed concomitantly for p-Akt/p-MAPK or pp-MAPK/Akt. (C) Optical density ratios (OD) of p-Akt levels in LN-18 and LN-229 cells were measured using ImageJ Software $1.37 \mathrm{v}$ (US National Institutes of Health). Ordinate units represent percent to the activated non-inhibited control. LY, LY294002; AEE, AEE788; AG, AG1478.

colony-forming potential was evaluated after a 10-day incubation (Fig. 5A and B). We chose to apply the inhibitor dosage of $5 \mu \mathrm{M}$ as it caused marked, yet not overshooting growth inhibition in both cell lines (Fig. 3). Furthermore, experiments using higher dosages of AEE788 revealed excessive cytotoxicity, leaving no space for evaluation of radiation-induced effects (Fig. 3A). A detailed analysis of the dose-response was conducted by fitting the survival curves into the linear-quadratic model (29) (Fig. 5C).

Both cell lines showed dose-dependent growth-inhibiting effects induced by irradiation with and without TKIs (Fig. 5). $\mathrm{D}_{37}$, the radiation dose reducing survival to $1 / \mathrm{e}=0.37$ of that
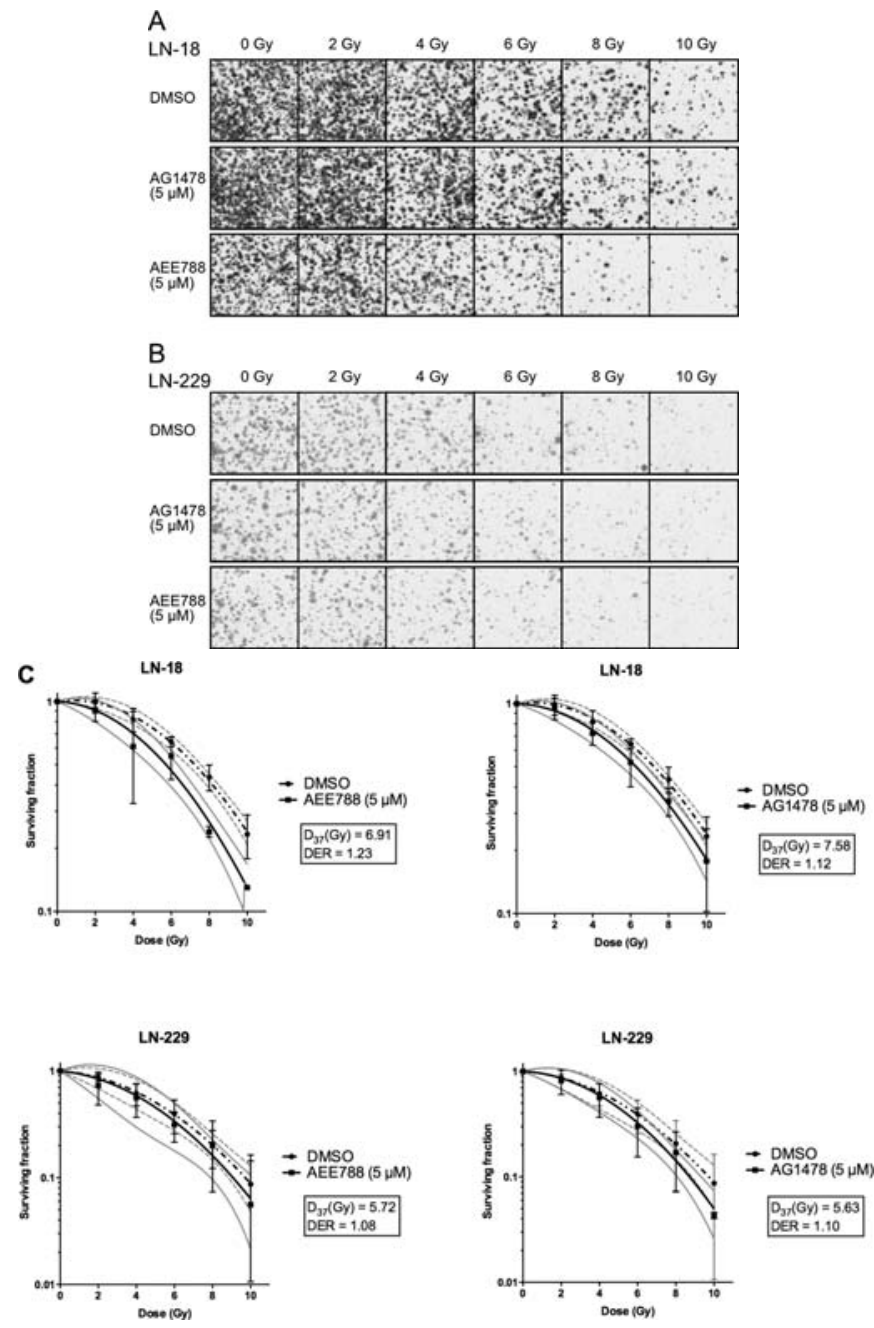

Figure 5. Dose-response clonogenic survival experiments in (A) LN-18 and (B) LN-229 cells, (C) analyzed using the linear-quadratic model. (A) LN-18 and (B) LN-229 cells were treated with $5 \mu \mathrm{M}$ AEE788 or $5 \mu \mathrm{M}$ AG1478 with or without concomitant $\gamma$-irradiation with single doses of 2, 4, 6, 8 or $10 \mathrm{~Gy}$. DMSO treated cells served as controls. (C) The surviving fraction (S) was fitted to the classical linear-quadratic equation, $S=\exp \left(-\alpha D-\beta D^{2}\right)$, where $\mathrm{D}$ is the radiation dose and $\alpha$ and $\beta$ adjustable parameters. $\mathrm{D}_{37}$ is the radiation dose resulting in $37 \%$ survival compared to non-irradiated controls. Doseenhancement ratio (DER) was calculated as the quotient of $\mathrm{D}_{37}$ (radiation alone) and $\mathrm{D}_{37}$ (radiation plus inhibitor). Points represent the average of three independent experiments and error bars depict the SD. Dotted lines represent the $95 \%$ confidence band of DMSO-survival curves and solid grey lines the 95\% confidence band of AG1478/AEE788-survival curves. Calculations were made using Graph Pad Prism software, version 5 (GraphPad Software Inc).

in controls, was computed for each survival curve, and the dose enhancement ratio (DER) for each inhibitor was acquired by comparison of $\mathrm{D}_{37}$ of TKI-treated cells to controls.

In irradiated LN-229 cells $\left(\mathrm{EGFR}^{+} / \mathrm{HER} 2^{++}\right)$only a trend toward increased growth inhibition after treatment with both EGFR and EGFR/HER2 inhibitors was discernable (DER >1), which did not reach statistical significance (overlapping 95\% confidence bands; Fig. 5C).

Conversely, in $\mathrm{LN}-18$ cells $\left(\mathrm{EGFR}^{++++} / \mathrm{HER} 2^{+}\right)$treated with $5 \mu \mathrm{M}$ AEE788, the DER was 1.23 and $\mathrm{D}_{37}$ differed to TKI-untreated controls on the 5\% niveau (non-overlapping 95\% confidence bands) starting at a dose of $6 \mathrm{~Gy}$. However, the sole EGFR inhibition failed to significantly reduce the radiation dose needed to reduce cell survival to $37 \%$ (Fig. 5C). 
In summary, this result indicates a moderate radiosensitising property of the bispecific EGFR/HER2 inhibitor AEE788 in the EGFR-overexpressing glioma cell line LN-18.

\section{Discussion}

Due to thus far disappointing results in the EGFR-targeted treatment of glioblastoma $(18,19)$, and considering the important ability for cross-talk between the members of the ErbB-receptor family (33), we aimed to evaluate the importance of HER2 signalling and concomitant EGFR/HER2 inhibition in this highly malignant neoplasm.

Using a model of two glioblastoma cell lines with an approximately inverse ratio of EGFR and HER2 together with wild-type PTEN status (22), we demonstrated a relative resistance of presumably EGFR-driven LN-18 cells $\left(\mathrm{EGFR}^{+++} / \mathrm{HER} 2^{+}\right)$to specific EGFR inhibition with AG1478. Interestingly, HER2-overexpressing LN-229 cells, harbouring only comparably low levels of EGFR $\left(\mathrm{EGFR}^{+} / \mathrm{HER}^{++}\right)$, reacted relatively sensitively to specific EGFR inhibition.

Of course, the complex nature of ErbB signalling precludes any rushed conclusion, and further studies are needed to verify the present results. Although our results at least presumably indicate that overexpression of EGFR in tumour cells expressing comparably less HER2 may not only fail to serve as a positive predictive marker for response to EGFR-targeted therapy, but may even render cells resistant to it. It may therefore be possible to explain why EGFR expression in various tumours has not proven to be a useful predictive marker to EGFR-targeted therapies in either preclinical studies, in which EGFR inhibition resulted in growth arrest of tumours expressing a wide range of EGFR levels $(34,35)$, or in clinical settings $(36,37)$. Conducting cellbased and xenograft experiments using the specific EGFRTKIs AG1478 or gefitinib, it was even suggested that HER2 overexpression indicates responsiveness to EGFR-targeted therapy (38-40). Our data are consistent with this observation, though we additionally point out that rather than total receptor amount, it may be the ratio of EGFR to HER2 that determines response to EGFR-targeted therapy. In support, a recently published corresponding observation revealed that the susceptibility of breast cancer cells to anti-HER2 targeting (using Trastuzumab) (to some extent) depends on the degree of EGFR co-expression rather than the individual HER2 amount (41).

Furthermore we showed some evidence that one mechanism for overcoming resistance to EGFR-targeted therapy might be to additionally target HER 2 with bispecific EGFR/HER2 TKIs. EGFR-overexpressing and HER2 coexpressing LN-18 cells (EGFR ${ }^{++++} / \mathrm{HER}^{+}$) showed a relative resistance to specific EGFR inhibition using AG1478, which could be overcome by AEE788, supposedly due to its bispecific EGFR and HER2-inhibiting activity.

These results somehow challenge the more orthodox interpretation of 'oncogenic addiction' (5-7), which focuses on one single target molecule. As they suggest, the sole blockade of the prominently overexpressed receptor might not be sufficient for interference with survival pathways. Focus is shifted to associated co-expressed molecules as important concomitant targets.
A conclusive hypothetical explanation for our observation could be the impaired EGFR/HER2 heterodimer activation and thus impaired downstream signalling induced by bispecific EGFR/HER2 inhibition in LN-18 cells and both EGFRspecific and bispecific EGFR/HER2 inhibition in LN-229 cells. Signalling through HER2 heterodimers is widely accepted to be superior in strength and duration over ErbB homodimer signalling (8), and EGFR/HER2 heterodimers are preferentially formed following EGF stimulation (31). This enhanced signalling potency and preferred formation have identified EGFR/HER2 heterodimers as one of the main effectors of the oncogenic potential of ErbB receptors, and thus pivotal for the proliferation of ErbB-dependent cancer cells and presumably important for TKI-induced growth inhibition.

Transferring this knowledge into a mechanistic explanation of the present findings, EGFR inhibition in EGFR-overexpressing $\mathrm{LN}-18$ cells $\left(\mathrm{EGFR}^{+++} / \mathrm{HER} 2^{+}\right.$) does not interfere with EGFR/HER2 heterodimer signalling, and hence induces no growth inhibiting effect. It is therefore implied that due to EGFR overexpression, the total amount of EGFR is only partially blocked by the specific EGFR TKI AG1478. Concomitant inhibition of the comparably lower amount of HER2, implying a complete receptor blockade due to the low receptor number, impairs heterodimer activation, which induces marked growth inhibition.

In LN-229 cells (EGFR ${ }^{+} / \mathrm{HER}^{++}$) harbouring comparably less EGFR while overexpressing HER2, sole EGFR inhibition is sufficient to inhibit activation of EGFR/HER2 heterodimers, a complete blockage of EGFR is implied by both EGFR and EGFR/HER2 TKIs.

In support of this hypothetical model and the importance of heterodimer signalling, recent clinical observations disclosed an adverse prognostic value of HER2 overexpression in breast cancer patients only when HER 2 was in the activated (i.e., phosphorylated) state or co-expressed with EGFR (42). A total of $97 \%$ of tumours expressing activated HER 2 coexpressed EGFR (42), highlighting the importance and prognostic value of ligand-dependent, i.e., ErbB dimerisation partner-dependent mechanisms of HER2 activation. In another clinical observation in patients with non-small cell lung cancer, sensitivity of EGFR-positive tumours treated with the specific EGFR-inhibitor gefitinib was associated with increased copy numbers of the HER2 gene (43).

Our data further indicate that together with MAPK inhibition, particularly inhibition of the PI3K/Akt pathway correlates with the growth suppressing effects of EGFR and EGFR/HER2 inhibition, suggesting that the PI3K/Akt pathway may be important in driving tumour growth in both EGFR and HER2-overexpressing GBM. Consistently, activation of the PI3K/Akt pathway has been found to be associated with reduced survival of glioma patients, and it is significantly more frequent in GBM than in non-GBM astrocytic tumours (44). We hypothesise that the relative resistance of LN-18 cells to sole EGFR inhibition may be partially caused by an incomplete blockage of Akt signalling, which can be overcome by additional inhibition of HER2. This claim is consistent with a recent clinical study disclosing Akt phosphorylation as the strongest predictor of a lack of response to EGFR-targeted therapy in GBM (45). 
Another propensity of ErbB receptors shows their inhibition to be especially valuable to amend radiotherapy treatment in GBM. It is known that ErbB receptors are activated by ionising radiation to produce radioprotective cellular responses, and thereby account for at least part of the radioresistance of cancer cells $(20,21)$. This leads to the assumption that inhibition of ErbB receptors with TKIs may prove to be a valuable approach to radiosensitising. Matching expectations, EGFR inhibitors have to date proven to exert some radiosensitising properties in preclinical and clinical settings in various solid tumours, even though controversial results have also been reported (46). In GBM cell-based studies, it was even shown that inhibition of the Akt signalling pathways with the PI3K inhibitor LY294002 mediates radiosensitisation (47). Unfortunately, however, LY294002 is not suitable for clinical use owing to its small therapeutic window (48), and EGFR-targeted therapy and irradiation in GBM patients to date has shown only disappointing clinical results $(19,49)$. This leads to the need to augment preclinical understanding of underlying principles of ErbB inhibitionmediated radiosensitisation.

We therefore aimed to evaluate the benefit of EGFR and HER2 inhibition following radiotherapy of glioma cells. Specific EGFR inhibition had no radiosensitising effect in EGFR-driven LN-18 cells $\left(\mathrm{EGFR}^{++++} / \mathrm{HER} 2^{+}\right)$or in HER2overexpressing LN-229 cells (EGFR+/HER $2^{++}$). Most interestingly though, the bispecific EGFR/HER2 inhibitor AEE788 showed a moderate propensity to radiosensitise LN-18 glioma cells, which overexpress EGFR and co-express HER2. This data further underline the potential of inhibition of HER2 and the impairment of EGFR/HER2 heterodimers in EGFR-driven tumours, a finding that may be of clinical significance and warrants further investigation. Although the dose enhancement ratio of AEE788 in LN-18 cells was only moderate in vitro $(\mathrm{DER}=1.23)$, it may well translate to substantial synergy in vivo, as reported in similar settings (50). Considering fractionated administration of radiation, the moderate DER of 1.23 per fraction (reported in this study) for each of, for example, 15 fractions would result in a substantial total enhancement ratio of $22.3\left(1.23^{15}\right)$. Additionally, effects on in vivo tumour microenvironments, such as angiogenesis, may further promote in vivo efficacy. ErbB tyrosine kinase inhibitors have been described to block the secretion of angiogenic factors, such as vascular endothelial growth factor (VEGF) and interleukin-8 (51), and AEE788 is known to exert direct anti-angiogenic activity through additional vascular endothelial growth factor receptor (VEGFR)-inhibition (25).

Considering the findings, the limitations of the present study should be kept in mind. Although the cell lines used presented a similar molecular status to some important molecules, e.g., PTEN, this does not exclude other molecular alterations influencing the response to the therapies applied. Despite the very low expression of VEGFR2 found by others and our group in preliminary Western blot analyses in both cell lines (data not shown) (52), it cannot be ruled out that some of the additional effects observed with AEE788 could be affected by VEGFR2 inhibition. Further studies using additional models, e.g., engineered cell lines, additional TKIs, direct receptor-binding studies and investigation regarding cell cycle, apoptosis and invasion/migration, should follow the present work in order to validate the presented hypothesis.

Especially in the context of the highly complex nature of ErbB receptor signalling, there is no doubt that the conclusion that HER2 expression may represent a mechanism of resistance to EGFR-targeted TKIs in glioblastoma may be a simplified approach. Mechanisms of resistance, and most importantly, mechanisms with the potential to overcome resistance to EGFR-TKI therapy, however, are desperately needed. In the present study, our data reveal the potential of HER2 heterodimer signalling to be a mechanism of resistance to EGFRtargeted TKIs. We believe this idea to be an important finding worth communicating.

It clearly cannot be assumed that results obtained on cell lines in vitro can be translated directly into the clinic. Nonetheless, preclinical studies arise as a sine qua non for successful clinical applications, providing insight into molecular characteristics that render patients susceptive to individualised and targeted therapy. We suggest that although EGFR TKIs are developed as 'targeted therapies' selective for EGFR-overexpressing tumours, there is evidence that their clinical testing should not be confined to patients with EGFRoverexpressing neoplasms.

In conclusion, the results of the present study propose the importance of EGFR/HER2 heterodimer receptor signalling in the EGFR-targeted therapy of glioblastoma. Our data indicate that resistance of EGFR-overexpressing tumours to selective EGFR inhibition (AG1478) may, to some extent, be overcome through AEE788, supposedly due to its concomitant HER2 inhibiting effect. In addition, we postulate that response to EGFR-targeted therapy may rather depend on the cellular ratio of EGFR to HER2 than on the total individual receptor amount. Further studies are needed to validate this hypothesis.

\section{Acknowledgements}

We are very grateful to Peter Traxler, Novartis AG, Basel, for supplying us with AEE788. We thank Guido Piontek (Department of Neuropathology, Institute of Pathology, Technical University of Munich, Munich, Germany) for expert technical assistance. This work was supported by the Deutsche Forschungsgemeinschaft (SFB 824: 'Imaging for the selection, monitoring and individualization of cancer therapies', Project B6).

\section{References}

1. Ohgaki H, Dessen P, Jourde B, et al: Genetic pathways to glioblastoma: a population-based study. Cancer Res 64: 6892-6899, 2004.

2. Ohgaki $\mathrm{H}$ and Kleihues P: Population-based studies on incidence, survival rates, and genetic alterations in astrocytic and oligodendroglial gliomas. J Neuropathol Exp Neurol 64: 479-489, 2005.

3. Stupp R, Mason WP, van den Bent MJ, et al: Radiotherapy plus concomitant and adjuvant temozolomide for glioblastoma. $\mathrm{N}$ Engl J Med 352: 987-996, 2005.

4. Kantarjian H, Sawyers C, Hochhaus A, et al: Hematologic and cytogenetic responses to imatinib mesylate in chronic myelogenous leukemia. N Engl J Med 346: 645-652, 2002.

5. Weinstein IB: Cancer. Addiction to oncogenes - the Achilles heal of cancer. Science 297: 63-64, 2002.

6. Weinstein IB and Joe A: Oncogene addiction. Cancer Res 68: 3077-3080, 2008 
7. Pillay V, Allaf L, Wilding AL, et al: The plasticity of oncogene addiction: implications for targeted therapies directed to receptor tyrosine kinases. Neoplasia 11: 448-458, 2009.

8. Yarden Y and Sliwkowski MX: Untangling the erbB signalling network. Nat Rev Mol Cell Biol 2: 127-137, 2001.

9. Ohgaki $\mathrm{H}$ and Kleihues P: Genetic pathways to primary and secondary glioblastoma. Am J Pathol 170: 1445-1453, 2007.

10. Citri A and Yarden Y: EGF-ERBB signalling: towards the systems level. Nat Rev Mol Cell Biol 7: 505-516, 2006.

11. Mineo JF, Bordron A, Baroncini M, et al: Low HER2-expressing glioblastomas are more often secondary to anaplastic transformation of low-grade glioma. J Neurooncol 85: 281-287, 2007.

12. Schwechheimer K, Laufle RM, Schmahl W, Knodlseder M, Fischer $\mathrm{H}$ and Hofler H: Expression of neu/c-ErbB-2 in human brain tumors. Hum Pathol 25: 772-780, 1994

13. Haynik DM, Roma AA and Prayson RA: HER-2/neu expression in glioblastoma multiforme. Appl Immunohistochem Mol Morphol 15: 56-58, 2007.

14. Engelhard HH, Wolters M and Criswell PS: Analysis of c-erbB2 protein content of human glioma cells and tumor tissue. J Neurooncol 23: 31-40, 1995.

15. Torp SH, Gulati S, Johannessen E and Dalen A: Coexpression of c-erbB 1-4 receptor proteins in human glioblastomas. An immunohistochemical study. J Exp Clin Cancer Res 26: 353-359, 2007.

16. Schlegel J, Stumm G, Brandle K, et al: Amplification and differential expression of members of the ErbB-gene family in human glioblastoma. J Neurooncol 22: 201-207, 1994.

17. Krause DS and Van Etten RA: Tyrosine kinases as targets for cancer therapy. N Engl J Med 353: 172-187, 2005.

18. Ziegler DS, Kung AL and Kieran MW: Anti-apoptosis mechanisms in malignant gliomas. J Clin Oncol 26: 493-500, 2008.

19. Brown PD, Krishnan S, Sarkaria JN, et al: Phase I/II trial of erlotinib and temozolomide with radiation therapy in the treatment of newly diagnosed glioblastoma multiforme: North Central Cancer Treatment Group Study N0177. J Clin Oncol 26: 5603-5609, 2008.

20. Contessa JN, Hampton J, Lammering G, et al: Ionizing radiation activates Erb-B receptor dependent Akt and p70 S6 kinase signaling in carcinoma cells. Oncogene 21: 4032-4041, 2002.

21. Nyati MK, Morgan MA, Feng FY and Lawrence TS: Integration of EGFR inhibitors with radiochemotherapy. Nat Rev Cancer 6: 876-885, 2006

22. Ishii N, Maier D, Merlo A, et al: Frequent co-alterations of TP53, p16/CDKN2A, p14ARF, PTEN tumor suppressor genes in human glioma cell lines. Brain Pathol 9: 469-479, 1999.

23. Diserens AC, de Tribolet N, Martin-Achard A, Gaide AC Schnegg JF and Carrel S: Characterization of an established human malignant glioma cell line: LN-18. Acta Neuropathol 53: $21-28,1981$.

24. Levitzki A and Gazit A: Tyrosine kinase inhibition: an approach to drug development. Science 267: 1782-1788, 1995

25. Traxler P, Allegrini PR, Brandt R, et al: AEE788: a dual family epidermal growth factor receptor/ErbB2 and vascular endothelial growth factor receptor tyrosine kinase inhibitor with antitumor and antiangiogenic activity. Cancer Res 64: 4931-4941, 2004.

26. Vlahos CJ, Matter WF, Hui KY and Brown RF: A specific inhibitor of phosphatidylinositol 3-kinase, 2-(4-morpholinyl)-8phenyl-4H-1-benzopyran-4-one (LY294002). J Biol Chem 269: 5241-5248, 1994.

27. Pang L, Sawada T, Decker SJ and Saltiel AR: Inhibition of MAP kinase kinase blocks the differentiation of PC-12 cells induced by nerve growth factor. J Biol Chem 270: 13585-13588, 1995.

28. Dudley DT, Pang L, Decker SJ, Bridges AJ and Saltiel AR: A synthetic inhibitor of the mitogen-activated protein kinase cascade. Proc Natl Acad Sci USA 92: 7686-7689, 1995.

29. Hall EJ: Cell survival curves. In: Radiobiology for the Radiologist. J.B. Lippincott Company, Philadelphia, 1994.

30. Dahle J, Kakar M, Steen HB and Kaalhus O: Automated counting of mammalian cell colonies by means of a flat bed scanner and image processing. Cytometry A 60: 182-188, 2004

31. Gulliford TJ, Huang GC, Ouyang X and Epstein RJ: Reduced ability of transforming growth factor-alpha to induce EGF receptor heterodimerization and downregulation suggests a mechanism of oncogenic synergy with ErbB2. Oncogene 15: 2219-2223, 1997.

32. Failly M, Korur S, Egler V, et al: Combination of sublethal concentrations of epidermal growth factor receptor inhibitor and microtubule stabilizer induces apoptosis of glioblastoma cells. Mol Cancer Ther 6: 773-781, 2007.
33. Gusterson BA and Hunter KD: Should we be surprised at the paucity of response to EGFR inhibitors? Lancet Oncol 10: 522-527, 2009

34. Sirotnak FM, Zakowski MF, Miller VA, Scher HI and Kris MG: Efficacy of cytotoxic agents against human tumor xenografts is markedly enhanced by coadministration of ZD1839 (Iressa), an inhibitor of EGFR tyrosine kinase. Clin Cancer Res 6: 4885-4892. 2000 .

35. Wakeling AE, Guy SP, Woodburn JR, et al: ZD1839 (Iressa): an orally active inhibitor of epidermal growth factor signaling with potential for cancer therapy. Cancer Res 62: 5749-5754, 2002.

36. Campiglio M, Locatelli A, Olgiati C, et al: Inhibition of proliferation and induction of apoptosis in breast cancer cells by the epidermal growth factor receptor (EGFR) tyrosine kinase inhibitor ZD1839 ('Iressa') is independent of EGFR expression level. J Cell Physiol 198: 259-268, 2004.

37. Johnson DH and Arteaga CL: Gefitinib in recurrent non-smallcell lung cancer: an IDEAL trial? J Clin Oncol 21: 2227-2229, 2003.

38. Emlet DR, Schwartz R, Brown KA, Pollice AA, Smith CA and Shackney SE: HER2 expression as a potential marker for response to therapy targeted to the EGFR. Br J Cancer 94: 1144-1153, 2006

39. Moulder SL, Yakes FM, Muthuswamy SK, Bianco R, Simpson JF and Arteaga CL: Epidermal growth factor receptor (HER1) tyrosine kinase inhibitor ZD1839 (Iressa) inhibits HER2/neu (erbB2)-overexpressing breast cancer cells in vitro and in vivo. Cancer Res 61: 8887-8895, 2001.

40. Moasser MM, Basso A, Averbuch SD and Rosen N: The tyrosine kinase inhibitor ZD1839 ('Iressa') inhibits HER2-driven signaling and suppresses the growth of HER2-overexpressing tumor cells. Cancer Res 61: 7184-7188, 2001

41. Brockhoff G, Heckel B, Schmidt-Bruecken E, et al: Differential impact of Cetuximab, Pertuzumab and Trastuzumab on BT474 and SK-BR-3 breast cancer cell proliferation. Cell Prolif 40: 488-507, 2007.

42. DiGiovanna MP, Stern DF, Edgerton SM, Whalen SG, Moore D 2nd and Thor AD: Relationship of epidermal growth factor receptor expression to ErbB-2 signaling activity and prognosis in breast cancer patients. J Clin Oncol 23: 1152-1160, 2005.

43. Cappuzzo F, Varella-Garcia M, Shigematsu H, et al: Increased HER2 gene copy number is associated with response to gefitinib therapy in epidermal growth factor receptor-positive nonsmall-cell lung cancer patients. J Clin Oncol 23: 5007-5018, 2005.

44. Chakravarti A, Zhai G, Suzuki Y, et al: The prognostic significance of phosphatidylinositol 3-kinase pathway activation in human gliomas. J Clin Oncol 22: 1926-1933, 2004.

45. Haas-Kogan DA, Prados MD, Tihan T, et al: Epidermal growth factor receptor, protein kinase B/Akt, and glioma response to erlotinib. J Natl Cancer Inst 97: 880-887, 2005.

46. Riesterer O, Milas L and Ang KK: Use of molecular biomarkers for predicting the response to radiotherapy with or without chemotherapy. J Clin Oncol 25: 4075-4083, 2007.

47. Nakamura JL, Karlsson A, Arvold ND, et al: PKB/Akt mediates radiosensitization by the signaling inhibitor LY294002 in human malignant gliomas. J Neurooncol 71: 215-222, 2005.

48. Riesterer O, Tenzer A, Zingg D, et al: Novel radiosensitizers for locally advanced epithelial tumors: inhibition of the PI3K/Akt survival pathway in tumor cells and in tumor-associated endothelial cells as a novel treatment strategy? Int J Radiat Oncol Biol Phys 58: 361-368, 2004.

49. Chakravarti A, Berkey B, Robins HI, et al: An update of phase II results from RTOG 0211: A phase I/II study of gefitinib + radiation for newly-diagnosed glioblastoma patients. Int J Radiat Oncol Biol Phys 66: S83-S84, 2006.

50. Nyati MK, Maheshwari D, Hanasoge S, et al: Radiosensitization by pan ErbB inhibitor CI-1033 in vitro and in vivo. Clin Cancer Res 10: 691-700, 2004.

51. De Luca A, Carotenuto A, Rachiglio A, et al: The role of the EGFR signaling in tumor microenvironment. J Cell Physiol 214: 559-567, 2008 .

52. Potapova O, Laird AD, Nannini MA, et al: Contribution of individual targets to the antitumor efficacy of the multitargeted receptor tyrosine kinase inhibitor SU11248. Mol Cancer Ther 5: 1280-1289, 2006 